\title{
Mating system and population genetic structure of an androdioecious tree, Fraxinus lanuginosa Koidz. (Oleaceae) in northern Japan
}

\author{
K Ishida ${ }^{1}$ and Tsutom Hiura ${ }^{2}$ \\ ${ }^{1}$ Kansai Research Center, Forestry and Forest Products Research Institute, Momoyama, Fushimi, Kyoto 612-0855, Japan; \\ ${ }^{2}$ Tomakomai Experimental Forest, Field Science Center for Northern Biosphere, Hokkaido University, Tomakomai 053-0035, Japan
}

\begin{abstract}
Models for the maintenance of androdioecy have suggested that selfing of hermaphrodites decreases the frequency of males in a population (the 'male frequency'). To test this hypothesis, we used electrophoretic allozyme methods to study the mating system and population genetics of an androdioecious tree, Fraxinus lanuginosa, which exhibits large variations in male frequency among subpopulations in central Hokkaido (northern Japan). We estimated the outcrossing rates by using seeds assayed at three polymorphic loci, and found that the multilocus outcrossing rate $\left(t_{\mathrm{m}}\right)$ increased with increasing male frequency $(q)\left(t_{m}=0.69\right.$ to 0.99 , $q=0.11$ to 0.59$)$. Fixation indices $\left(F_{j}\right)$ estimated from these $t_{m}$ values ranged from 0.006 to 0.185 , and were significantly greater than zero in plots with a low male frequency $(q \leq 0.35)$, but not in plots with intermediate or high male frequencies $(q \geq 0.47)$. However, fixation indices at the late
\end{abstract}

life stage $\left(F_{a}\right)$ were not significantly different from zero at five loci $\left(F_{a}=0.056\right.$ to $0.101, q=0.11$ to 0.61$)$, and the $F_{a}$ values were lower than the $F_{j}$ values in several of the plots where both values were measured. These results indicate that inbreeding depression substantially decreases the proportion of selfed progeny in partially self-fertilising subpopulations. A theoretical model for the maintenance of androdioecy showed expected male frequencies significantly lower than the observed values in plots with high male frequency ( $q=0.59$ to 0.61 ), although the differences between the expected and observed values of male frequencies were not significant in plots with intermediate or low male-frequencies $(q=0.11$ to 0.47$)$. In this paper, the factors are discussed that affect variations in the male frequency among subpopulations of $F$. lanuginosa based on these findings. Heredity (2002) 88, 296-301. DOI: 10.1038/sj/hdy/6800043

Keywords: androdioecy; mating system; population genetic structure; male frequency; Fraxinus lanuginosa

\section{Introduction}

Androdioecy is a rare reproductive system in which male plants coexist with plants that have perfect flowers or cosexual plants (plants that bear both male and female flowers) in a breeding population (Yampolsky and Yampolsky, 1922). In this paper, we have chosen the term 'hermaphrodite' to represent plants with perfect flowers. Most supposedly androdioecious species are functionally dioecious or monoecious, and only seven species have been reported to exhibit true functional androdioecy (Charlesworth, 1984; Vassiliadis et al, 2000a). According to phenotypic selection models (Lloyd, 1975; Charlesworth and Charlesworth, 1978), the rarity of functional androdioecy results from the need for high pollen fecundity in the male plants. For male plants to be maintained in a population, the pollen fecundity of these plants must be at least double that of hermaphroditic plants (Lloyd, 1975). However, if male fertility depends on the male frequency in a population, the requirements

Correspondence: K Ishida, Kansai Research Center, Forestry and Forest Products Research Institute, Momoyama, Fushimi, Kyoto 612-0855, Japan.E-mail: ishidak@affrc.go.jp

Received 22 May 2001; accepted 15 October 2001 of a phenotypic selection model may be relaxed (Vassiliadis et al, 2000a).

Phenotypic selection models predict that, in androdioecious plants, males must have a greater fecundity to be maintained in partially selfing populations than in outbreeding populations, because fewer ovules are available for outcrossing (Lloyd, 1975; Charlesworth and Charlesworth, 1978). Thus, the models predict that male frequency decreases in an androdioecious population with increasing selfing rate when the relative (male vs hermaphrodite) pollen fecundity is constant, since selfing decreases the relative fitness of males in this condition unless the male frequency decreases. A metapopulation dynamics model also predicts that self-incompatible populations maintain a higher frequency of male plants than do self-compatible populations (Pannell, 1997a). Because all androdioecious species show variations in male frequency between populations (eg, Liston et al, 1990; Pannell, 1997b; Akimoto et al, 1999), and self-compatibility, except for Phillyrea angustifolia (Vassiliadis et al, $2000 \mathrm{~b}$ ), studies of the relationship between the magnitude of inbreeding and male frequency provide a way to test these theoretical predictions.

However, few studies have measured both male frequency and the magnitude of inbreeding in androdioecious species. In Datisca glomerata, outcrossing rates are 
sufficiently high to satisfy the theoretical requirements, and the observed values of male frequency were similar to the expected values in two populations (Fritsch and Rieseberg, 1992). On the other hand, the inbreeding coefficients in Schizopepon bryoniaefolius are so high that androdioecy in this species seems to contradict the theoretical predictions for the maintenance of androdioecy (Akimoto et al, 1999).

Fraxinus lanuginosa Koidz. (Oleaceae), a sub-canopy tree species (Hiura et al, 1998) distributed in Japan and in Korea, is functionally androdioecious and is pollinated by both insects and wind (Ishida and Hiura, 1994, 1998). Males produce pollen that is more than eight times as fertile as that of hermaphrodites, which suggests that androdioecy of the species is maintained by the high pollen fecundity of the males compared with that of the hermaphrodites (Ishida and Hiura, 1998). In central Hokkaido (northern Japan), the species exhibits large variations in male frequency among subpopulations; thus, these subpopulations provide an opportunity to test the prediction that inbreeding affects the maintenance of androdioecy. The mating system of this species has not been fully studied, and thus it is not clear whether hermaphrodites partially self-fertilize under natural conditions, although manual pollination experiments have shown that hermaphrodites exhibit self-sterility (Ishida and Hiura, 1998).

In this paper, we attempt to resolve the relationship between the magnitude of inbreeding and male frequency in F. lanuginosa. We also ask whether the nature of androdioecy in this species supports theoretical predictions for the maintenance of androdioecy. To accomplish this goal, the mating system was studied (the outcrossing rate at the seed stage) and the population genetic structures (heterozygosity and fixation index at the late life stage) of the species using electrophoretic allozyme methods. The theoretical predictions of a phenotypic selection model were tested (Lloyd, 1975) by using the estimated mating parameters and published data on the species.

\section{Materials and methods}

\section{Study sites}

Our study sites were located in the Tomakomai Experimental Forest of Hokkaido University (lat. $42^{\circ} 40^{\prime} \mathrm{N}$, long. $141^{\circ} 37^{\prime} \mathrm{E}, 40-80 \mathrm{~m}$ alt.) and in a neighbouring national forest; these broad-leaved natural forests have Quercus crispula, Acer mono, Ostrya japonica, and Tilia japonica as the dominant tree species (Hiura et al, 1998). Five study plots were set up in 1993 and another five in 1997, and measured the frequency of flowering trees, the male frequency, and the growth rate of F. lanuginosa trees with girth at breast height (gbh) greater than $12 \mathrm{~cm}$. Seeds were collected to estimate the outcrossing rates in 1997 at four of the 10 plots, since not enough seeds could be collected for this analysis in the other six plots (Table 1). The population genetics in five of the ten plots were examined, whose male frequencies included the highest and lowest values in the original 10 plots.

\section{Electrophoretic analysis}

The outcrossing rates were estimated at the seed stage using between 143 and 254 seeds collected from eight or

nine randomly chosen trees in each of the four plots (12

to 29 seeds per tree; Table 1). The population genetic parameters (heterozygosity, F-statistics, and fixation index) at the late life stage (tree height $>1 \mathrm{~m}$ ) were also measured by using winter buds from randomly chosen trees in five plots (19 to 60 trees per plot). Seed and bud materials were stored at $5^{\circ} \mathrm{C}$ before analysis by electrophoresis. For electrophoresis of the seed materials, embryos from the seeds were ground in $150 \mu \mathrm{l}$ of cold extraction buffer (modified based on the methods of Shiraishi, 1988). For bud materials, leaf primordia from the buds were ground in $500 \mu l$ of the cold extraction buffer.

The following five enzymes were analysed: alcohol dehydrogenase (ADH; EC 1. 1. 1. 1), fluorescent esterase (FE; EC 3. 1. 1. 1), leucine amino peptidase (LAP; EC 3. 4. 11. 1), phosphoglucose isomerase (PGI; EC 5.3.1.9), and 6-phosphogluconate dehydrogenase (6PGD; EC 1.1.1.44). The standard abbreviation of E.C. number 3. 1. 1. 1. is esterase (EST), but there are alternative staining methods for the enzyme (Richardson et al, 1986), and thus it has been denoted as fluorescent esterase in the case of a staining method with methylumbelliferyl acetate and UV light. Starch gels $(12 \% \mathrm{w} / \mathrm{v})$ were used to resolve ADH, FE, and PGI, and polyacrylamide vertical slab gels were used for LAP and 6PGD. For the starch gels, gel and electrode buffer system number 8 described by Soltis et al (1983) was applied, and electrophoresis was conducted following the general methods of Gottlieb (1981a) and Soltis et al (1983). For the polyacrylamide vertical slab gels, the gels were prepared according to the methods of Davis (1964) and Orstein (1964), and electrophoresis was carried out at $4^{\circ} \mathrm{C}$ with a current of $12.3 \mathrm{~mA} / \mathrm{cm}^{2}$ for $150 \mathrm{~min}$.

Genetic interpretations of the resulting banding patterns were inferred from segregation patterns with reference to typical subunit structures (Gottlieb, 1981b, 1982; Crawford, 1983). In the analysis of the seed materials, $\mathrm{ADH}$ was not scored for four plots because of poor activity, and FE and LAP were not scored for plots 1 through 3 and plot 4, respectively, because of poor resolution. Three polymorphic loci were then used to estimate the outcrossing rates at the seed stage (Lap-1, Pgi, and $6 P g d$ for plots 1 through 3 , and Fe, Pgi, and $6 P g d$ for plot $4)$, and five loci were used for the population genetic parameters at the late life stage (Adh, Fe, Lap-1, Lap-2, and $6 P g d)$.

The multilocus $\left(t_{m}\right)$ was calculated and mean singlelocus $\left(t_{s}\right)$ outcrossing rates at the seed stage by using the MLT program of Ritland (1990a). MLT is based on the multilocus outcrossing estimation procedure of Ritland and Jain (1981), which assumes that progeny are derived from either random mating (outcrossing) or self-fertilisation. The difference between the two values $\left(t_{m}-t_{s}\right)$ indicates the magnitude of the biparental inbreeding. The 95\% bootstrap confidence intervals for the outcrossing rates were calculated based on 1000 bootstrap samples (resampling of seeds within each family) by using the method of Manly (1997). The fixation index $\left(F_{j}\right.$; Wright, 1969) was also estimated using the $t_{m}$ value, by assuming that no inbreeding depression occurred after the seed stage. The $F_{j}$ value is a measure of the magnitude of inbreeding, and is calculated from the following equation (modified from Falconer and Mackay, 1996):

$$
F_{j}=\left(1-t_{m}\right) /\left(1+t_{m}\right) .
$$


Table 1 Plot area, male frequency, density and mean size of flowering trees, and number of samples examined for allozyme analysis in each Fraxinus lanuginosa plot. Male frequencies were measured based on five years of observation (Hiura and Ishida, unpublished data). The density and mean size of the flowering trees were measured in 1997

\begin{tabular}{|c|c|c|c|c|c|c|c|c|}
\hline \multirow[t]{2}{*}{ Plot no. } & \multirow{2}{*}{$\begin{array}{l}\text { Plot area } \\
\left(m^{2}\right)\end{array}$} & \multicolumn{2}{|c|}{ Male frequency } & \multirow{2}{*}{$\begin{array}{l}\text { Tree density } \\
\quad(\text { no./ha) }\end{array}$} & \multicolumn{2}{|c|}{ Girth at breast height $(\mathrm{cm})^{c}$} & \multicolumn{2}{|c|}{ No. of samples } \\
\hline & & Frequency $^{a}$ & Category $^{b}$ & & Males & Hermaphrodites & Seeds ${ }^{d}$ & Trees \\
\hline 1 & 3200 & $0.59(51)$ & $\mathrm{H}$ & 159.4 & $39.2 \pm 16.6$ & $50.0 \pm 16.3$ & $143(8)$ & 60 \\
\hline 2 & 2400 & $0.61(64)$ & $\mathrm{H}$ & 266.7 & $36.8 \pm 14.6$ & $40.5 \pm 15.6$ & - & 60 \\
\hline 3 & 6400 & $0.47(75)$ & I & 117.2 & $33.4 \pm 9.0$ & $31.1 \pm 12.0$ & 179 (9) & 60 \\
\hline 4 & 6400 & $0.25(8)$ & $\mathrm{L}$ & 9.4 & $31.8 \pm 12.3$ & $41.0 \pm 17.1$ & - & 27 \\
\hline 5 & 2400 & $0.11(9)$ & $\mathrm{L}$ & 33.3 & 45.4 & $39.4 \pm 4.8$ & 254 (9) & 19 \\
\hline 10 & 2000 & 0.35 (17) & $\mathrm{L}$ & 85.0 & $36.5 \pm 10.7$ & $37.5 \pm 18.5$ & $157(8)$ & - \\
\hline
\end{tabular}

a Values in parentheses indicate the number of flowering trees observed from 1993 to $1997 .{ }^{b} \mathrm{H}=$ high male frequency; $\mathrm{I}=$ intermediate male frequency; $\mathrm{L}=$ low male frequency. These categories were determined based on the first and third quartiles of data from 10 plots, which include the male frequencies for the six plots in this table and four other plots in the study area (Hiura and Ishida, unpublished data). ${ }^{c}$ Mean \pm standard error. ${ }^{\mathrm{d}}$ Values in parentheses indicate the number of maternal trees.

The $95 \%$ bootstrap confidence intervals for the $F_{j}$ values were calculated based on the 1000 bootstrap samples for the $t_{m}$ values.

The observed and expected values of heterozygosity were calculated at the late life stage by using the Biosys1 program of Swofford and Selander (1989). The expected heterozygosity represents the value expected under a Hardy-Weinberg equilibrium, and measures the magnitude of genetic diversity within a population. F-statistics $\left(F_{i s}, F_{s t}\right.$, and $F_{i t} ;$ Wright, 1969; Weir and Cockerham, 1984) at the late life stage were then calculated by using the FSTAT program of Goudet (1995). F-statistics provide a set of measures of the heterozygote deficiency compared with values in a Hardy-Weinberg equilibrium; respectively, $F_{i s}, F_{s t}$, and $F_{i t}$ measure the heterozygote deficit within a population, the heterozygote deficit among populations, and the global deficit in all populations. The 95\% confidence intervals for the F-statistics were calculated by bootstrapping over the aforementioned loci.

We also calculated the fixation index at the late life stage $\left(F_{a}\right)$ by using FSTAT. The $F_{a}$ value measures the heterozygote deficiency compared with a Hardy-Weinberg equilibrium at the late life stage for each population. If there is no inbreeding depression at the late life stage, the $F_{a}$ value is identical to the $F_{j}$ value. The $F_{a}$ values were then tested for deviation from a Hardy-Weinberg equilibrium by using a 5000-example randomisation (ie, the Hardy-Weinberg equilibrium was generated by randomising alleles among individuals within a population).

\section{Results}

\section{Outcrossing rate and male frequency}

The $t_{m}$ and $t_{s}$ values for the four plots ranged from 0.69 to 0.99 and from 0.66 to 0.99 , respectively, and both parameters increased with increasing male frequency (Table 2). As the rank of male frequency was equal to that of tree density for each of the four plots, the $t_{m}$ and $t_{s}$ values also increased with increasing tree density (Table 1). There was significant correlation between the rank of male frequency and that of tree density for the six plots (Spearman's $\rho=0.94, P<0.005$, Table 1 ).

The differences between the $t_{m}$ and $t_{s}$ values were close to zero $\left(t_{m}-t_{s}<0.03\right)$, indicating that biparental inbreeding was not significant in the four plots. The $t_{m}$ and $t_{s}$ values for plots 5 and 10, which exhibited the lowest male frequencies $(q=0.11$ and 0.35 , respectively), were lower than 0.9 and differed significantly from $1(P<0.05$; significance determined by the upper limit of the $95 \%$ bootstrap confidence interval). In contrast, the values for the other two plots, which exhibited intermediate or high male frequency $(q=0.59$ and 0.47 , respectively), were higher than 0.9 and did not differ significantly from 1 $(P>0.05)$. These results indicate that hermaphroditic trees partially self-fertilise in plots with low male frequency, whereas trees predominantly outcross in plots with intermediate or high male frequency. The fixation indices $\left(F_{j}\right)$ estimated from the $t_{m}$ values ranged from 0.006 to 0.185 in the four plots; these estimates were significantly higher than 0 in plots with low male frequency, but not in plots with intermediate or high male frequency $(P>0.05$; significance was determined by using the lower limit of the $95 \%$ bootstrap confidence interval).

\section{Population genetic structure at the late life stage}

The observed and expected heterozygosity values for all loci (Table 3) for the five plots ranged from 0.22 to 0.34 and from 0.21 to 0.35 , respectively, and there was no significant correlation between the expected heterozygosity and male frequency (Spearman's $\rho=0.10, P>0.05$ ). $F_{i s \prime}$ $F_{s t}$ and $F_{i t}$ for the five populations were 0.02 (95\% confidence interval from -0.06 to 0.08 ), 0.19 (from 0.08 to 0.30 ), and 0.20 (from 0.04 to 0.31 ), respectively, indicating that most of the heterozygote deficiency compared with a Hardy-Weinberg equilibrium arose from the Wahlund effect (ie, the effect of subdividing a population on the heterozygote deficiency) rather than from the mating system.

The fixation indices $F_{a}$ for all loci in the five plots (Table 3 ) ranged from -0.056 to 0.101 , and did not differ significantly from 0 (based on a randomisation test; $P>0.05)$. There was no significant correlation between the $F_{a}$ values and male frequency (Spearman's $\rho=0.10$, $P>0.05)$. The $F_{a}$ estimates were lower than the $F_{j}$ estimates in three of the five plots (plots 1, 3, and 5) for which both values were measured (Tables 2 and 3).

\section{Discussion}

Mating system and population genetic structure

Estimation of the outcrossing rate revealed that $F$. lanuginosa predominantly exhibits random mating (out- 
Table 2 Multilocus $\left(t_{m}\right)$ and mean single-locus $\left(t_{s}\right)$ outcrossing rates, and the inbreeding coefficients $\left(F_{j}\right)$ estimated from outcrossing rates in Fraxinus lanuginosa. The $95 \%$ confidence intervals appear in parentheses

\begin{tabular}{|c|c|c|c|c|c|}
\hline Plot no. & Rank of male frequency ${ }^{a}$ & $t_{m}$ & $t_{s}$ & $t_{m}-t_{s}$ & $F_{j}$ \\
\hline 1 & 1 & $0.99(0.83-1.18)$ & 0.99 (0.81-1.13) & $0.000(-0.06-0.10)$ & $0.006(-0.078-0.092)$ \\
\hline 3 & 2 & $0.93(0.78-1.07)$ & $0.92(0.78-1.04)$ & $0.002(-0.05-0.08)$ & $0.039(-0.031-0.124)$ \\
\hline 5 & 4 & $0.69(0.55-0.84)$ & $0.66(0.53-0.79)$ & $0.031(-0.04-0.05)$ & $0.185(0.086-0.292)$ \\
\hline 10 & 3 & $0.83(0.67-0.94)$ & $0.82(0.64-0.92)$ & $0.019(-0.03-0.08)$ & $0.091(0.029-0.199)$ \\
\hline
\end{tabular}

aThe ranks of the male frequency values were determined based on the male frequency values reported in Table 1.

Table 3 Heterozygosity values and fixation index $\left(F_{a}\right)$ at the late life stage for five loci in Fraxinus lanuginosa. Standard errors appear in parentheses

\begin{tabular}{lcccr}
\hline Plot no. & Rank of male frequency & Observed heterozygosity & Expected heterozygosity & Fixation index $\left(F_{a}\right)$ \\
\hline 1 & 2 & $0.243(0.067)$ & $0.238(0.071)$ & -0.047 \\
2 & 1 & $0.280(0.054)$ & $0.310(0.058)$ & 0.101 \\
3 & 3 & $0.327(0.040)$ & $0.350(0.051)$ & 0.030 \\
4 & 4 & $0.221(0.079)$ & $0.210(0.078)$ & -0.056 \\
5 & 5 & $0.341(0.062)$ & $0.325(0.058)$ & -0.050 \\
\hline
\end{tabular}

aThe ranks of the male frequency values were determined based on the male frequency values reported in Table 1.

crossing) on sites in central Hokkaido with high male frequencies, but partially self-fertilises on sites with low male frequencies. The inbreeding found in the present study is not due to biparental inbreeding, since this form of inbreeding appears insignificant in the species $\left(t_{m}-t_{s}\right.$ was not significantly different from 0; Table 2). This result demonstrates that $F$. lanuginosa does not exhibit complete self-incompatibility, and that self-fertilisation of the species is facultative. This contradicts previous studies that have shown the species to be self-sterile in the study area.

Facultative selfing has been reported in other species that exhibit self-incompatibility or self-sterility (eg, Bertin et al, 1989; Becerra and Lloyd, 1992). For example, Phormium tenax exhibits cryptic self-incompatibility, but selffertilises when cross-pollination is limited (Becerra and Lloyd, 1992). In the same manner, the self-fertilisation of $F$. lanuginosa that occurred on sites with low male frequency may have resulted from the limited cross-pollination, because male frequency is correlated to tree density in this species (Table 1; Hiura and Ishida, unpublished data). Thus, the amount of cross-pollination would decrease as male frequency decreases, and the mating system of the species appears to be density-dependent. Another androdioecious plant, Datisca glomerata, also appears to have a density-dependent outcrossing rate (Fritsch and Rieseberg, 1992). The low pollen fecundity of $F$. lanuginosa hermaphrodites compared with that of males (Ishida and Hiura, 1998) may also limit cross-pollination on sites with low male frequency, since the amount of pollen production per tree increases with increasing male frequency.

A possible alternative cause of the variation in outcrossing rates involves the dissolution of self-incompatibility in part of the population. However, this explanation did not seem to apply in the present study of $F$. lanuginosa. This is because the dissolution of self-incompatibility may result from the genetic bottleneck (Reinartz and Les, 1994) accompanied by a low level of heterozygosity, whereas the level of heterozygosity of $F$. lanuginosa in the present study was so high that this possibility can be ruled out.

The fixation indices $\left(F_{a}\right)$ at the late life stage did not differ significantly from 0 , and the $F_{i s}$ value was also close to 0 . Moreover, the $F_{a}$ values were lower than the fixation indices $\left(F_{j}\right)$ estimated from the $t_{m}$ values. These results indicate that inbreeding depression substantially decreases the proportion of selfed progeny. This is because inbreeding depression generally decreases the fixation index (inbreeding coefficient) over the course of the plant's life (Ritland, 1990b), and the $F_{a}$ value of zero requires a condition that no selfed progenies survive to the late life stage. This would lead to predominant outbreeding even in partially self-fertilising subpopulations. This result is consistent with the argument that predominantly outcrossing species (with an outcrossing rate $>0.6$ ) would exhibit strong inbreeding depression (Husband and Schemske, 1996), and with the prediction that populations of long-lived plants would have a large genetic load (Klekowski, 1988).

\section{Maintenance of androdioecy in $F$. lanuginosa}

Does androdioecy in F. lanuginosa support the theoretical predictions required for the maintenance of androdioecy? We tested the predictions of the phenotypic selection models of Lloyd (1975) and Charlesworth and Charlesworth (1978). The expected value of male frequency given by these models is:

$$
q=[t-2 l v(t+i-t i)] /[2 i v(1-t)(1-l)+t(1+v-2 l v)],
$$

where $t$ is the primary outcrossing rate, $i$ is the relative fitness (selfed/crossed) of selfed seeds $((1-i)$ equals the magnitude of the inbreeding depression), $l$ is the relative pollen fecundity (hermaphrodite/male), and $v$ is the relative viability of zygotes (hermaphrodite/male) up to reproductive maturity (Lloyd, 1975).

Thus, the observed and expected values of male frequency in four plots (plots 1,3,5, and 10) can be compared by inserting the data into the equation, along with hypothetical values for $i$ (Figure 1). It is assumed that 


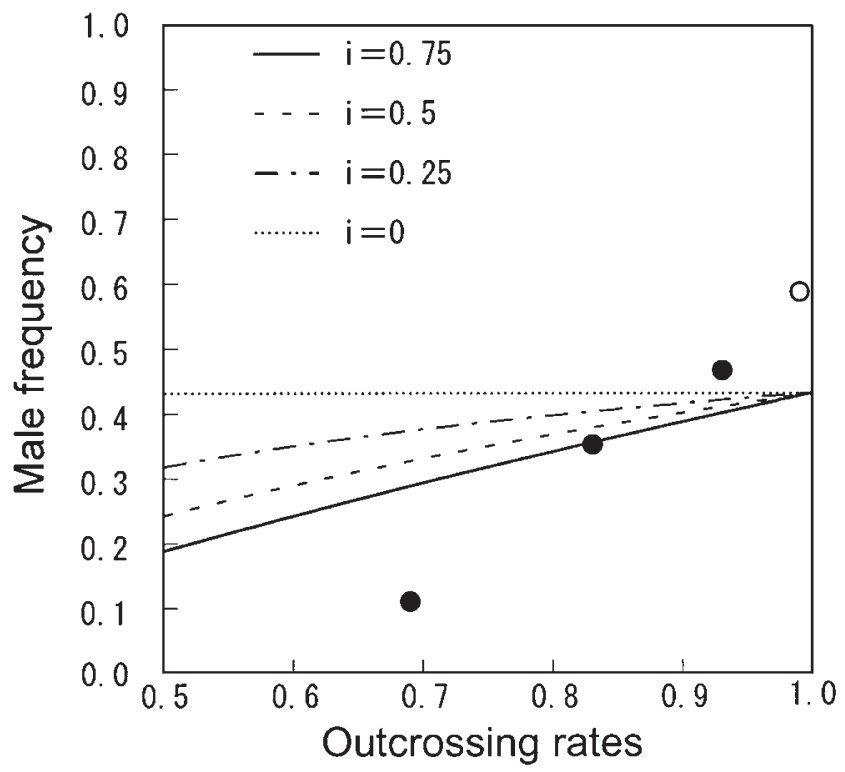

Figure 1 The relationship between the male frequencies and the outcrossing rates in Fraxinus lanuginosa. The lines show the expected values based on the model presented by Lloyd (1975). The parameter $i$ indicates the relative fitness (selfed/crossed) of selfed seeds. Solid circles represent the observed values of male frequency that did not differ significantly from the expected values (Fisher's exact test; $P>0.05$ ); the open circle represents an observed value that differed significantly from the expected value (Fisher's exact test; $P<0.05$ ).

$v=1.0$, because there were no significant differences in size structure (frequency distribution of gbh) and growth rates between males and hermaphrodites in the study area (Hiura and Ishida, unpublished data). When a value of 0.12 is substituted for $l$ (Ishida and Hiura, 1998), and hypothetical values $(0,0.25,0.5$, and 0.75$)$ for $i$, the model shows that the observed value was significantly higher than the expected values for plot 1 , which exhibited the highest male frequency among the four plots (observed $q=0.59)$, although the difference between the observed and expected values of male frequency was not significant in the other three plots.

If the observed and expected values using the six plots are compared, by assuming that the two remaining plots (plots 2 and 4) had one of the four estimated outcrossing values, then the model again shows similar results; the observed values were significantly higher than the expected values in the plots with high male frequency (plots 1 and 2; observed $q=0.59$ and 0.61, respectively), whereas the difference between the observed and expected values was not significant in the other four plots, which exhibited intermediate or low male frequency (observed $q$ ranged from 0.11 to 0.47 ). These results indicate that the high male frequencies observed in at least two plots cannot be explained by phenotypic selection models, and understanding the maintenance of androdioecy in $F$. lanuginosa requires an alternative theory.

In six other androdioecious species, the observed data for two species are inconsistent with the phenotypic selection models; in Schizopepon bryoniaefolius, the inbreeding coefficients in androdioecious populations are so high that the maintenance of androdioecy in the species contradicts the models (Akimoto et al, 1999). In Philly- rea angustifolia, several populations exhibit male frequencies close to 0.5 , a value that cannot be explained by the phenotypic selection models (Vassiliadis et al, 2000a), although its male frequencies in stressed sites seem to be explained by the models (Pannell and Ojeda, 2000). Vassiliadis et al (2000a) have proposed an alternative model for the maintenance of androdioecy that would explain high male frequencies that cannot be explained by the phenotypic selection models. They showed that if a gametophytic self-incompatibility locus is linked to a nuclear sex-determination locus, frequency-dependent selection caused by this linkage can result in higher male frequencies than the expected values based on the phenotypic selection model. If the self-sterility of $F$. lanuginosa arises from an incomplete gametophytic self-incompatibility linked with a sex-determination locus, then the proposed alternative model can be applied to the high male frequencies of F. lanuginosa, although the number of Salleles must be known before the model can be tested. Moreover, it is likely that other mechanisms, such as the plasticity of pollination by insects with respect to flower density, cause frequency-dependent selection. This plasticity of pollination would lead to a high male frequency in F. lanuginosa. To test these hypotheses would require studies of the mechanism of self-sterility and of the pollination biology of the species.

A possible alternative cause of the inconsistency between the observed and expected values for male frequency is the existence of sex-labile trees. In F. lanuginosa, sex-labile trees were found during 5 years of observation in central Hokkaido (three of 354 trees (0.8\%); Hiura and Ishida, unpublished data). If a sex-labile tree is considered to be functionally cosexual in terms of its genetic contribution to the next generation, the frequencies of functional males would be lower than the highest putative male frequency based on short-term observations; that is, the frequency of functional males would be lower than the highest expected male frequency (0.43). Although the frequency of the sex-labile trees found by Hiura and Ishida was small, the frequency of sex-labile trees in the longer term may be a significant factor in determining male frequency, because the typical life span of F. lanuginosa is long (about 100 years; Miyajima, 1979). Sex-labile individuals and an environmentally determined sex ratio have been found in another androdioecious plant, Mercurialis annua (Pannell, 1997a, c). This final hypothesis will be tested by conducting both a long-term survey of sex expression in F. lanuginosa and a study of the genetic mechanisms of sex determination.

\section{Acknowledgements}

We thank Kyoko Tanaka and Yukiko Sakamoto for assistance in laboratory work. We also thank Maki Masayuki, Hisashi Satoh, and two anonymous reviewers for their invaluable comments on the manuscript. This work was partly supported by grants to Tsutom Hiura from the Ministry of Education, Science, Sports and Culture of Japan (grants 07760141, 08406011, 09NP1501, 11440223).

\section{References}

Akimoto J, Fukuhara T, Kikuzawa K (1999). Sex ratios and genetic variation in a functionally androdioecious species, Schizopepon bryoniaefolius (Cucurbitaceae). Am J Bot 86: 880-886. 
Becerra JX, Lloyd DG (1992). Competition-dependent abscission of self-pollinated flowers of Phormium tenax (Agavaceae): a second action of self-incompatibility at the whole flower level? Evolution 46: 458-469.

Bertin RI, Barnes C, Guttman SI (1989). Self-sterility and cryptic self-fertility in Campsis radicans (Bignoniaceae). Bot Gaz 150: 397-403.

Charlesworth D (1984). Androdioecy and the evolution of dioecy. Biol J Linn Soc 22: 333-348.

Charlesworth B, Charlesworth D (1978). A model for the evolution of dioecy and gynodioecy. Am Nat 112: 975-997.

Crawford DJ (1983). Phylogenetic and systematic inferences from electrophoretic studies. In: Tanksley SD, Orton TJ (eds) Isozymes in Plant Genetics and Breeding, Part A, Elsevier: Amsterdam. pp 257-287.

Davis BJ (1964). Disk electrophoresis II: method and application to human serum proteins. Ann NY Acad Sci 121: 404-427.

Falconer DS, Mackay TFC (1996). Introduction to Quantitative Genetics, 4th edn. Longman Group: Edinburgh.

Fritsch P, Rieseberg LH (1992). High outcrossing rates maintain male and hermaphrodite individuals in populations of the flowering plant Datisca glomerata. Nature 359: 633-636.

Gottlieb LD (1981a). Gene number in species of Astereae that have different chromosome numbers. Proc Natl Acad USA 78 3726-3729.

Gottlieb LD (1981b). Electrophoretic evidence and plant populations. In: Reinhold L, Harborne JB, Swain T (eds) Progress in Phytochemistry, vol. 7, Pergamon: New York. pp 1-46.

Gottlieb LD (1982). Conservation and duplication of isozymes in plants. Science 216: 373-380.

Goudet J (1995). FSTAT (vers. 1.2): a computer program to calculate F-statistics. J Hered 86: 485-486.

Hiura T, Fujito E, Ishii T, Naniwa A, Sugata S, Ishida $\mathrm{K}$ et al (1998). Stand structure of a deciduous broad-leaved forest in Tomakomai Experimental Forest, based on a large-plot data. Res Bull Coll Exp For Fac Agric Hokkaido Univ 55: 1-10. (in Japanese, with English summary).

Husband BC, Schemske DW (1996). Evolution of the magnitude and timing of inbreeding depression in plants. Evolution $\mathbf{5 0}$ 54-70.

Ishida K, Hiura T (1994). Reproductive ecology in Fraxinus lanuginosa (II), pollen vector. Trans Hokkaido Br Jpn For Soc 42: 6163. (in Japanese).

Ishida K, Hiura T (1998). Flowering phenology and pollen fertility in an androdioecious tree, Fraxinus lanuginosa Koidz. (Oleaceae), in Hokkaido, Japan. Int J Pl Sci 159: 941-947.

Klekowski EJ Jr (1988). Genetic load and its causes in long-lived plants. Trees 2: 195-203.

Liston A, Rieseberg LH, Elias TS (1990). Functional androdioecy in the flowering plant Datisca glomerata. Nature 343: 641-642.

Lloyd DG (1975). The maintenance of gynodioecy and androdioecy in angiosperms. Genetica 45: 325-339.

Manly BFJ (1997). Randomization, Bootstrap and Monte Carlo Methods in Biology. Second edn. Chapman \& Hall: London.
Miyajima H (1979). Growth and wood quality of Japanese ash tree grown in Hidaka District, Hokkaido. Res Bull Exp For Hokkaido Univ 36: 421-450.

Orstein I (1964). Disk electrophoresis I: background and theory. Ann NY Acad Sci 121: 321-349.

Pannell JR (1997a). Widespread functional androdioecy in Mercurialis annua L. (Euphorbiaceae). Biol J Linn Soc 61: 95116.

Pannell JR (1997b). The maintenance of gynodioecy and androdioecy in a metapopulation. Evolution 51: 10-20.

Pannell JR (1997c). Mixed genetic and environmental sex determination in an androdioecious population of Mercurialis annua L. (Euphorbiaceae). Heredity 78: 50-56.

Pannell JR, Ojeda F (2000). Patterns of flowering and sex-ratio variation in the Mediterranean shrub Phillyrea angustifolia (Oleaceae): implications for the maintenance of males with hermaphrodites. Ecol Lett 3: 495-502.

Reinartz JA, Les DH (1994). Bottleneck-induced dissolution of self-incompatibility and breeding system consequences in Aster furcatus (Asteraceae). Am J Bot 81: 446-455.

Richardson BJ, Bavertock PR, Adams M (1986). Allozyme Electrophoresis. Academic Press: San Diego.

Ritland K (1990a). A series of FORTRAN computer programs for estimating plant mating systems. J Hered 81: 235-237.

Ritland K (1990b). Inferences about inbreeding depression based on changes of the inbreeding coefficient. Evolution 44: 12301241.

Ritland K, Jain S (1981). A model for the estimation of outcrossing rate and gene frequencies using $n$ independent loci. Heredity 47: 35-52.

Shiraishi S (1988). Inheritance of isozyme variations in Japanese Black Pine, Pinus thunbergii Parl. Silvae Genet 37: 93-100.

Soltis DE, Haufler CH, Darrow DC, Gastony GJ (1983). Starch gel electrophoresis of ferns: a compilation of grinding buffers, gel and electrode buffers, and staining schedules. Am Fern J 73: 9-27.

Swofford DL, Selander RB (1989). BIOSYS-1 A computer program for the analysis of allelic variation in population genetics and biochemical systematics. Release 1.7. University of Illinois: Urbana, Illinois, USA.

Vassiliadis C, Valero M, Saumitou-Laprade P, Godelle B (2000a). A model for the evolution of high frequencies of males in an androdioecious plant based on a cross-compatibility advantage of males. Heredity 85: 413-422.

Vassiliadis C, Lepart J, Saumitou-Laprade P, Vernet P (2000b). Self-incompatibility and male fertilization success in Phillyrea angustifolia (Oleaceae). Int J Pl Sci 161: 393-402.

Weir BS, Cockerham CC (1984). Estimating F-statistics for the analysis of population structure. Evolution 38: 1358-1370.

Wright S (1969). The Theory of Gene Frequencies, Evolution and the Genetics of Populations. Vol. 2. University of Chicago Press: Chicago.

Yampolsky C, Yampolsky H (1922). Distribution of sex forms in the phanerogamic flora. Bibl Genet 3: 1-62. 